\title{
Deuterated polycyclic aromatic hydrocarbons: Revisited
}

\author{
K. D. Doney ${ }^{1}$, A. Candian ${ }^{1}$, T. Mori ${ }^{2}$, T. Onaka ${ }^{2}$, and A. G. G. M. Tielens ${ }^{1}$ \\ ${ }^{1}$ Leiden Observatory, University of Leiden, PO Box 9513, 2300 RA Leiden, The Netherlands \\ e-mail: doney@strw. leidenuniv.nl \\ 2 Department of Astronomy, Graduate School of Science, The University of Tokyo, 7-3-1 Hongo, Bunkyo-ku, 113-0033 Tokyo, Japan
}

Received 23 June 2015 / Accepted 1 December 2015

\section{ABSTRACT}

\begin{abstract}
Aims. The amount of deuterium locked up in polycyclic aromatic hydrocarbons (PAHs) has to date been an uncertain value. We present a near-infrared (NIR) spectroscopic survey of HII regions in the Milky Way, Large Magellanic Cloud, and Small Magellanic Cloud obtained with AKARI, which aims to search for features indicative of deuterated PAHs (PAD or $\mathrm{D}_{n}$-PAH) to better constrain the $\mathrm{D} / \mathrm{H}$ ratio of PAHs.

Methods. Fifty-three HII regions were observed in the NIR $(2.5-5 \mu \mathrm{m})$, using the Infrared Camera (IRC) on board the AKARI satellite. Through comparison of the observed spectra with a theoretical model of deuterated PAH vibrational modes, the aromatic and (a)symmetric aliphatic C-D stretch modes were identified.

Results. We see emission features between 4.4-4.8 $\mu \mathrm{m}$, which could be unambiguously attributed to deuterated PAHs in only six of the observed sources, all of which are located in the Milky Way. In all cases, the aromatic C-D stretching feature is weaker than the aliphatic C-D stretching feature, and, in the case of M17b, this feature is not observed at all. Based on the weak or absent PAD features in most of the observed spectra, it is suggested that the mechanism for PAH deuteration in the ISM is uncommon.
\end{abstract}

Key words. astrochemistry - HII regions - infrared: ISM

\section{Introduction}

All deuterium (D; heavy hydrogen) was formed during the Big Bang and has subsequently been depleted through the process of astration, i.e., destruction by nuclear reactions in the interior of stars (Epstein et al. 1976; Linsky et al. 2006). As a result, the amount of deuterium in a galaxy, either as a free atom in the gas phase or locked up in molecules or grains, provides a direct measure of cosmic nucleosynthesis and is related to the chemical evolution of the galaxy itself. Of particular interest is the incorporation of deuterium in polycyclic aromatic hydrocarbon $(\mathrm{PAH})$ molecules. These molecules are ubiquitous and abundant in the interstellar medium (ISM); their UV/visible-pumped fluorescence is almost universally believed to give rise to aromatic infrared bands (AIBs). These bands dominate the mid-infrared (MIR) spectra of many interstellar objects, such as HII regions, planetary, and reflection nebulae, the diffuse ISM, and even entire galaxies (Leger \& Puget 1984; Allamandola et al. 1985, 1989; Tielens 2008; Joblin \& Tielens 2011; Li \& Draine 2012). The large heat capacity and aromaticity (Schutte et al. 1993) of PAHs suggests that once formed in stellar ejecta (Frenklach \& Feigelson 1989; Cherchneff et al. 1992) the PAH molecule is stable against photodissociation, at least compared to other ISM molecules. However, studies of meteorites (Kerridge et al. 1987 ) and recent laboratory experiments (Sandford et al. 2000; Thrower et al. 2012; Klærke et al. 2013) show that PAHs can undergo processing, such as oxidation, reduction, and isotope exchange, which depend on the environments the PAH experience. The large size ( $>50 \mathrm{C}$ atoms) and numerous hydrogen atoms of interstellar PAHs allows them to potentially be a large reservoir of deuterium in the ISM (Allamandola et al. 1989; Tielens 1992; Hudgins et al. 2004). Consequently, deuteriumenriched PAHs have been suggested as a possible explanation for the variation of the gas phase atomic $\mathrm{D} / \mathrm{H}$ ratio in the intermediate region of the Milky Way galaxy, which has an average value of $\sim 14 \mathrm{ppm}$, but has a range of a factor of 4-5 with measurements as low as about $5.0 \mathrm{ppm}(\theta$ Car) and as high as about $21.8 \mathrm{ppm}\left(\gamma^{2} \mathrm{Vel}\right)$; the $17 \mathrm{ppm}$ depletion in some regions cannot be explained through astration alone (Peeters et al. 2004; Draine 2004; Linsky et al. 2006; Onaka et al. 2014).

Deuterium atoms can replace hydrogen atom in PAHs and can participate in the same characteristic vibrational modes (Bauschlicher et al. 1997). The heavier mass of deuterium shifts the $\mathrm{C}-\mathrm{D}$ vibrational modes to longer wavelengths relative to the corresponding $\mathrm{C}-\mathrm{H}$ vibrational modes (Bauschlicher et al. 1997; Allamandola 1993; Sandford et al. 2000; Hudgins et al. 2004). Hydrogen or deuterium when bonded to the carbon skeleton such that the carbon retains its $\mathrm{sp}^{2}$ hybridization (denoted PAH or PAD, respectively) results in the aromatic C-H stretch mode at $3.29 \mu \mathrm{m}$ (Allamandola et al. 1989; Sandford et al. 2013) or the aromatic C-D stretch mode at $4.40 \mu \mathrm{m}$ (Bauschlicher et al. 1997; Hudgins et al. 2004). If the hydrogen or deuterium adds to the $\mathrm{PAH}$, resulting in a carbon with $\mathrm{sp}^{3}$ hybridization (denoted $\mathrm{H}_{n}-\mathrm{PAH}$ or $\mathrm{D}_{n}-\mathrm{PAH}$, respectively), then the $\mathrm{CH}_{2}$ or $\mathrm{CDH}$ groups show both asymmetric and symmetric aliphatic stretching modes. These features fall at 3.40 and $3.50 \mu \mathrm{m}$ for the $\mathrm{C}-\mathrm{H}$ asymmetric and symmetric stretching modes, respectively, (Sandford et al. 2013), and 4.63 and $4.75 \mu \mathrm{m}$ for the C-D asymmetric and symmetric stretching modes (Hudgins et al. 2004). Recently, Buragohain et al. (2015) showed that the $4.75 \mu \mathrm{m}$ feature may also be due to the C-D stretch of deuteronated PAH cations $\left(\mathrm{D}_{n}-\mathrm{PAH}^{+}\right)$. For both $\mathrm{C}-\mathrm{H}$ and $\mathrm{C}-\mathrm{D}$ aliphatic stretching modes, the intensity of the asymmetric stretch is intrinsically greater than the symmetric stretch because of the larger dipole moment. Other infrared features indicative of deuterated PAHs can arise in the $9-18 \mu \mathrm{m}$ as 
a result of bending of the C-D bonds. However, their exact position will vary across that region depending on the number of nonadjacent D atoms (Peeters et al. 2004), in some cases superimposing on the $\mathrm{C}-\mathrm{H}$ bending modes of standard PAHs.

Detection of $\mathrm{PAD} / \mathrm{D}_{n}$-PAHs features in the IR spectrum present the astronomer with several challenges. All three of the deuterated $\mathrm{C}-\mathrm{H}$ features are predicted to be weak (Bauschlicher et al. 1997; Hudgins et al. 2004). Also, their expected positions coincide with $\mathrm{HI}$ emission lines, and the symmetric stretching feature at $4.75 \mu \mathrm{m}$ is the only feature to lie in a portion of the spectrum clear of other emission lines. Additionally, groundbased observations of deuterated PAHs are almost impeded by the absorption band of telluric $\mathrm{CO}_{2}$ between $4.3-4.7 \mu \mathrm{m}$ (Bauschlicher et al. 1997; Hudgins et al. 2004). The best targets for the search of deuterated PAHs are regions where the PAH emission is strongest, such as the surface layers of photodissociation regions (PDRs) in HII regions.

So far, deuterated PAHs have been detected by ISO-SWS in the Orion Bar and M17 primarily through the C-D asymmetric stretching feature. The estimated number of deuterium atoms relative to hydrogen atoms on PAHs (denoted PAH D/H), based on the ratio of sum of the 4.4 and $4.63 \mu \mathrm{m}$ intensities to the 3.29 and 3.4-3.5 $\mu \mathrm{m}$ intensities, were found to be $0.17 \pm 0.03$ in the Orion Bar, and $0.36 \pm 0.08$ in M17 (Peeters et al. 2004) without considering the intrinsic intensities between the C-D and C-H stretching features, a factor of $\sim 1.75$ (Bauschlicher et al. 1997). The $\mathrm{PAH} \mathrm{D} / \mathrm{H}$ ratio, based on deuterated $\mathrm{PAH}$ features in M17, was indeed consistent with the observed Galactic variation of atomic D/H ratio in the gas phase. Recently, Onaka et al. (2014) reported an upper limit PAH D/H ratio of $0.029 \pm 0.002$ in the Orion Bar and $0.023 \pm 0.004$ in M17 with AKARI observations at slightly different pointing positions compared to the ISO-SWS observations, and taking the intrinsic intensities into account. The significant difference in the observed deuterium abundance in PAHs made it desirable to obtain multiple spectra of a large number of sources, at a variety of galactic evolution stages, with high signal-to-noise ratios (S/Ns) to better constrain the deuterium abundance in PAHs, and to determine if the deuterium fractionation of PAHs could be not only highly variable between sources, but also within a source. To this end, we present our search for deuterated PAHs in a sample of HII regions observed with AKARI.

This article is organized as follows. Section 2 contains the details of the NIR spectroscopic observations of 53 HII regions using the AKARI satellite and necessary data reduction method. Section 3 discusses the spectral results, including the first detection of the deuterated PAHs in six Galactic sources, and in Sects. 4 and 5 the astrophysical implications and conclusion are presented.

\section{Observations and data reduction}

The infrared camera (IRC) of the AKARI satellite offers NIR spectroscopy of the $2.5-5 \mu \mathrm{m}$ region with a resolution of $R \sim 100$ for diffuse sources (Onaka et al. 2007). The observations in this study were taken during the post-helium mission phase (Phase 3$)$ of the AKARI satellite with the Nh slit $\left(1^{\prime} \times 3^{\prime \prime}\right)$ with grism disperser, providing a dispersion of $0.0097 \mu \mathrm{m} / \mathrm{px}$ in this range (Onaka et al. 2007). At $5 \mu \mathrm{m}$ the grism sensitivity decreases compared to $3 \mu \mathrm{m}$ region, resulting in a larger noise level in the region where the PAD features are expected.

This study is based on the DABUN observational program, which observed seven HII regions in the Large Magellanic Cloud
(LMC), five in the Small Magellanic Cloud (SMC), and eight in the Milky Way (MW), chosen based on their corresponding radio data (Paladini et al. 2003; Filipovic et al. 1998, respectively). Thirty-three additional Milky Way sources were added from the AKARI Near-Infrared Spectral Atlas of Galactic HII regions version 1 public release (Mori et al. 2014). The details of the observation data are given in Table A.1.

The data reduction was carried out with the official AKARI spectroscopy pipeline for the Phase 3 data (version 20111121; Onaka et al. 2009). Spectra were extracted from the area corresponding to the brightest PAH flux at $3.3 \mu \mathrm{m}$. This corresponds to extraction areas of $10.22 \times 3^{\prime \prime}$ for LMC and SMC sources, 7 !' $3 \times 3^{\prime \prime}$ or 10 .' $22 \times 3^{\prime \prime}$ for MW sources that were part of the DABUN program and $8 . .76 \times 3^{\prime \prime}$ for MW sources retrieved from the AKARI public release catalog of Mori et al. (2014). The resulting spectra were subsequently spatially and spectrally smoothed by 3 pixels to remove shot noise without significantly changing the spatial or spectral resolution. In later observations, the thermal noise was noticeable even after pipeline processing, requiring additional post-pipeline dark current subtraction, which was performed following the procedure outlined by Mori et al. (2014).

For most of the targets, we took two or three observations (Table A.1). The pointing accuracy of AKARI can vary up to about $30^{\prime \prime}$ between the intended and actual target pointing positions; as such we analyzed each observation separately. In the cases with three observations of the same source, the spectrum with significantly different features was removed from analysis based on the assumption that, because of limited pointing accuracy, that spectrum was observing a significantly different part of the HII region. The selection was further reduced by removing the spectra without sufficient $\mathrm{S} / \mathrm{N}$ to quantify extinction or PAH emission features.

\section{Results and analysis}

All of the analyzed spectra show a number of features, typical of $\mathrm{HII}$ regions, such as $\mathrm{HI}$ recombination lines, $\mathrm{CO}_{2}$ ice features, and the PAH bands at 3.29 and 3.4-3.6 $\mu \mathrm{m}$ (Fig. 1). The uncertainty in the relative flux calibration is less than $10 \%$. The MW sources have better $\mathrm{S} / \mathrm{N}$ compared to the LMC and SMC sources, which lie on average at distances of $\sim 50 \mathrm{kpc}$ (Pietrzyński et al. 2013) and $\sim 60 \mathrm{kpc}$ (Hilditch et al. 2005), respectively. As a result, while the PAH aromatic $\mathrm{C}-\mathrm{H}$ stretching mode at $3.29 \mu \mathrm{m}$ is seen in all of the spectra, in some of the LMC and SMC spectra the PAH flux is too weak to distinguish the aliphatic $\mathrm{C}-\mathrm{H}$ features from the noise.

Some of the MW source spectra show ice absorption features of $\mathrm{H}_{2} \mathrm{O}$ and $\mathrm{CO}_{2}$, which likely arise from cold interstellar clouds between the source and AKARI. All of the obtained spectra show emission features indicative of ionized gas in HII regions. For example, the prominent $\mathrm{H}$ I recombination lines $\mathrm{Br} \alpha$ at $4.052 \mu \mathrm{m}$ and $\operatorname{Br} \beta$ at $2.626 \mu \mathrm{m}$ are seen in all spectra, and a few also show a number of other hydrogen and helium recombination lines, all of which are fit with Gaussian functions; the fit parameters are listed in Table 1 . There is a shift in the observed central wavelength of the $\mathrm{H}$ I lines relative to their literature values, but the discrepancy is within the uncertainty of the wavelength calibration of $\sim 0.005 \mu \mathrm{m}$.

The continuum is fit with a 3 rd order polynomial, taking into account the broad continuum plateau from 3.2-3.6 $\mu \mathrm{m}$, and then subtracted. The $\mathrm{H}_{2} \mathrm{O}$ absorption feature around $3.05 \mu \mathrm{m}$ is fit via laboratory spectrum of pure $\mathrm{H}_{2} \mathrm{O}$ ice at $10 \mathrm{~K}$ taken 
K. D. Doney et al.: Deuterated polycyclic aromatic hydrocarbons: Revisited
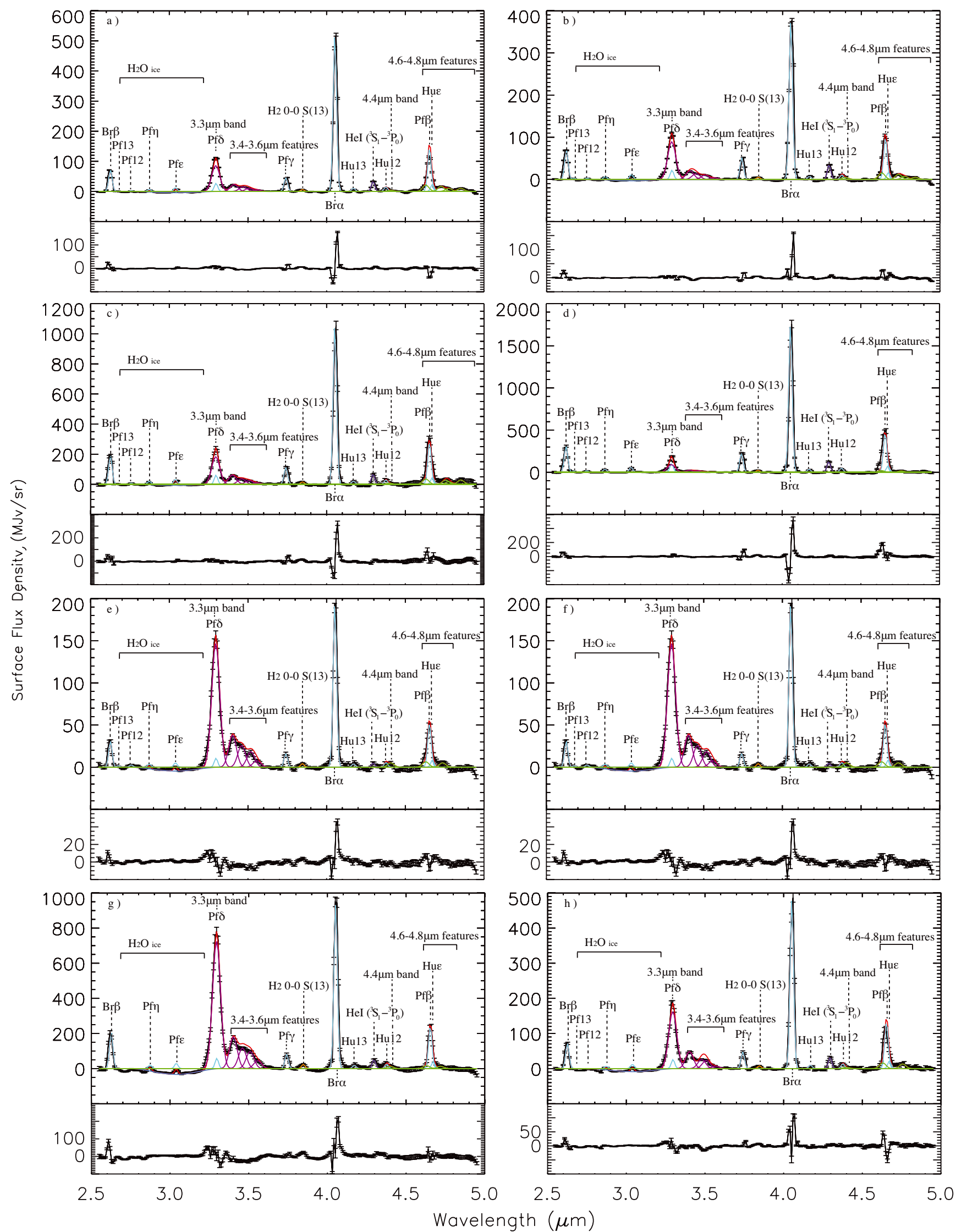

Fig. 1. Fitting results for the spectra of a) IRAS 12073-6233 obs. 1; b) IRAS 12073-6233 obs. 2; c) NGC 3603; d) M17b; e) W51 obs. 1; f) W51 obs. 2; g) M8; and h) G75.783+0.343 in red. The black line is the observed spectra, the H I emission lines are fit in cyan, the HeI emission line is fit in navy, the $\mathrm{H}_{2} \mathrm{O}$ ice absorption line is fit in blue, the $\mathrm{H}_{2}$ rotational line is fit in orange, $\mathrm{PAH}$ and $\mathrm{H}_{n}$ - $\mathrm{PAH}$ features are fit in purple, and PAD and $\mathrm{D}_{n}$-PAH features are fit in green. Below each figure is the corresponding residual plot. 
Table 1. Gaussian profile parameters for ice absorption features and emission lines fitted in the spectra.

\begin{tabular}{|c|c|c|}
\hline Line & $\lambda_{\text {center }}(\mu \mathrm{m})$ & $F W H M(\mu \mathrm{m})$ \\
\hline $\mathrm{HI} \mathrm{Br} \beta$ & 2.6259 & 0.026 \\
\hline HI Pf13 & 2.6751 & 0.026 \\
\hline HI Pf12 & 2.7583 & 0.026 \\
\hline $\mathrm{HI} \mathrm{Pf} \eta$ & 2.8730 & 0.026 \\
\hline $\mathrm{HI} \operatorname{Pf} \epsilon$ & 3.0392 & 0.026 \\
\hline PAH & 3.29 & 0.060 \\
\hline $\mathrm{HI} \operatorname{Pf} \delta$ & 3.2970 & 0.026 \\
\hline $\mathrm{H}_{n}-\mathrm{PAH}$ & 3.40 & 0.058 \\
\hline $\mathrm{H}_{n}-\mathrm{PAH}$ & 3.45 & 0.058 \\
\hline $\mathrm{H}_{n}-\mathrm{PAH}$ & 3.50 & 0.058 \\
\hline $\mathrm{H}_{n}-\mathrm{PAH}$ & 3.56 & 0.058 \\
\hline HI Pf $\gamma$ & 3.7406 & 0.026 \\
\hline $\mathrm{H}_{2} 0-0 \mathrm{~S}(13)$ & 3.846 & 0.026 \\
\hline $\mathrm{HI} \mathrm{Br} \alpha$ & 4.0523 & 0.026 \\
\hline HI Hu13 & 4.1708 & 0.026 \\
\hline $\mathrm{CO}_{2}$ & 4.26 & 0.047 \\
\hline $\mathrm{He} \mathrm{I}\left({ }^{3} \mathrm{~S}_{1}-{ }^{3} \mathrm{P}_{0}\right)$ & 4.2954 & 0.026 \\
\hline HI Hu12 & 4.3765 & 0.026 \\
\hline PAD & 4.40 & 0.047 \\
\hline $\mathrm{D}_{n}-\mathrm{PAH}$ & 4.63 & 0.047 \\
\hline HI Pf $\beta$ & 4.6538 & 0.026 \\
\hline $\mathrm{HI} \mathrm{Hu} \epsilon$ & 4.6725 & 0.026 \\
\hline $\mathrm{D}_{n}-\mathrm{PAH}$ & 4.75 & 0.047 \\
\hline $\mathrm{D}_{n}-\mathrm{PAH}$ & 4.80 & 0.047 \\
\hline $\mathrm{D}_{n}-\mathrm{PAH}$ & 4.85 & 0.047 \\
\hline
\end{tabular}

from the Leiden Ice Database (Gerakines et al. 1996). In contrast, the $\mathrm{CO}_{2}$ ice feature cannot be completely resolved using the AKARI/IRC slit spectroscopy, and is fit using a negative Gaussian function; details of the fit for the spectra are listed in Table 1.

The PAH and $\mathrm{D}_{n}$-PAH fluxes at 3.29 and $4.63 \mu \mathrm{m}$, respectively, overlap with the $\mathrm{HI}$ emission lines $\operatorname{Pf} \delta$ at $3.297 \mu \mathrm{m}, \operatorname{Pf} \beta$ at $4.654 \mu \mathrm{m}$, and $\mathrm{Hu} \epsilon$ at $4.673 \mu \mathrm{m}$. The expected HI emission intensities are determined relative to $\operatorname{Br} \alpha$ and $\operatorname{Br} \beta$, assuming case B recombination conditions of $T_{\mathrm{e}}=10^{4} \mathrm{~K}$ and $n_{\mathrm{e}}=10^{4} \mathrm{~cm}^{-3}$ for the Milky Way and $T_{\mathrm{e}}=10^{4} \mathrm{~K}$ and $n_{\mathrm{e}}=10^{2} \mathrm{~cm}^{-3}$ for the LMC and SMC (Storey \& Hummer 1995) and an extinction law of $A_{\lambda}=\lambda^{-1.7}$ (Martín-Hernández et al. 2002a). To determine the flux of the underlying features, the overlapping HI lines are modeled as Gaussian functions and subtracted from the spectrum. The extinction-corrected intensity subtraction introduces an uncertainty of $\sim 10 \%$ to the flux of the underlying feature. The observed flux at 4.17 and $4.37 \mu \mathrm{m}$ can be fit by the HI Hu13 and Hu12 expected flux, with some excess flux at $4.4 \mu \mathrm{m}$.

The PAH and $\mathrm{H}_{n}-\mathrm{PAH}$ features and their deuterium counterparts are fit with Gaussian functions; the details of the fits are given in Table 1. Based on band position coincidence between observed excess flux and laboratory (Sandford et al. 2000) and theoretical data (Hudgins et al. 2004), PAD and/or $\mathrm{D}_{n}-\mathrm{PAH}$ features were detected in six Milky Way sources: IRAS 120736233, NGC 3603, M8, M17b, W51, and G75.783+0.343 (Figs. 1 and 2); the calculated PAH and $\mathrm{H}_{n}$-PAH fluxes are summarized in Table A.2, while the calculated PAD and $\mathrm{D}_{n}$-PAH fluxes are summarized in Table A.3. Of the six sources, IRAS 12073-6233 and W51 had two observations that showed PAD and $\mathrm{D}_{n}-\mathrm{PAH}$ features. In addition three sources: IRAS 15384-5348, M17a, and NGC 3576, show a less than $3 \sigma$ detection of the asymmetric C-D stretch, but because of noisy baseline the accompanying symmetric mode was not seen. Aromatic C-H stretch overtone and combination bands, as well as, contributions from aliphatic side groups also fall in the range of $3.4-3.6 \mu \mathrm{m}$
(Allamandola et al. 1989; Sandford et al. 2013; Pilleri et al. 2015), and similarly, contributions from the C-D analogs likely fall between $4.6-5.0 \mu \mathrm{m}$. For analysis, we assume that aliphatic groups are attached to the main PAH rings, i.e., superhydrogenated PAHs/PADs contribute the majority of the flux in these wavelength ranges, and the determined strength of these bands are consequently upper limits.

The aromatic C-D stretch feature at $4.4 \mu \mathrm{m}$ and aliphatic C-D (a)symmetric stretch features at $4.63-4.85 \mu \mathrm{m}$ are present in all eight of the spectra (Figs. 1 and 2), with the exception of M17b. As noted by Peeters et al. (2004) for M17, the nearby source M17b does not show the aromatic C-D stretch band. The aromatic C-D stretch mode is observed to be blended with the HI Hu12 emission line. Nonetheless, two Gaussian profiles are needed to reproduce the observed spectrum, and detections of the same blended feature in multiple sources suggest that the feature is not noise even though they are only $\sim 1 \sigma$ level detections. The aliphatic C-D asymmetric stretch feature is heavily blended with $\mathrm{HI}$ emission lines and, as a result, its intensity is an estimation based on the intrinsic intensity ratio of the C-D symmetric to asymmetric modes and the intensity of the unobscured C-D symmetric feature. Simultaneous fitting of the $\operatorname{Pf} \beta, \operatorname{Hu} \epsilon$, and estimated C-D asymmetric stretch features is able to reproduce the observed spectrum, which suggests that the estimation is good. In the case of IRAS 12073-6233 and NGC 3603 (Figs. 1a), b), c) and Figs. 2a), b), c)), the PAD and $\mathrm{D}_{n}-\mathrm{PAH} \mathrm{S} / \mathrm{N}$ was large enough that the minor features seen in the Hudgins et al. (2004) modeled spectra at 4.8-4.9 $\mu \mathrm{m}$ are discernible. In NGC 3603, however, the minor feature at $4.84 \mu \mathrm{m}$ has a significantly larger intensity than expected based on the model in Hudgins et al. (2004), which is likely a result of the continuum subtraction.

In the 3.8-4.6 $\mu \mathrm{m}$ region, there are a number of features that can be attributed to either HI lines, the 0-0 S(13)-S(9) rovibrational transitions of $\mathrm{H}_{2}$, or deuterated PAHs. Notably, the $\mathrm{S}(10)$ transition of $\mathrm{H}_{2}$ at $4.4 \mu \mathrm{m}$ overlaps the expected position of the aromatic C-D stretch mode. The $\mathrm{S}(13)$ transition at $3.85 \mu \mathrm{m}$ is seen clearly in all eight of the spectra. Based on the intensities of the excess flux at the positions corresponding to the $\mathrm{S}(12)-\mathrm{S}(9)$ transitions of $\mathrm{H}_{2}$ and level populations predicted by non-LTE models of $\mathrm{H}_{2}$ (Bertoldi et al. 2000), we cannot rule out the $\mathrm{S}(10)$ transition of $\mathrm{H}_{2}$ as the carrier of the $4.4 \mu \mathrm{m}$ excess flux at the present spectral resolution. However, for the analysis we assume the $4.4 \mu \mathrm{m}$ feature is the aromatic C-D stretch in order to obtain an upper limit on its abundance. In the case of the C-D aliphatic stretch features, the excess fluxes at $4.63 \mu \mathrm{m}$ and $4.75 \mu \mathrm{m}$ do not match the $\mathrm{S}(9)$ transition of $\mathrm{H}_{2}$ within the wavelength calibration uncertainties, and thus the $\mathrm{H}_{2}$ transition does not influence their assignments.

If we only take the intrinsic intensity ratio of C-D to C-H features into account, which reduces the overall intensities for deuterium features by a factor of $\sim 1.75$ (Bauschlicher et al. 1997), the number of deuterium atoms relative to hydrogen atoms on PAHs is then estimated from the ratio of the sum of the corrected deuterated features to the sum of the hydrogenated features. An observed upper limit of the $\mathrm{PAH} \mathrm{D} / \mathrm{H}$ is determined to be between 0.03 and 0.44 , which is summarized in Table A. $3^{1}$. For sources with multiple observations, W51 and IRAS 12073-6233, it was found that the PAH

\footnotetext{
1 The given PAH D/H uncertainties in Table A.3 do not take errors of various origins into account, including the difference of excitation of PADs/ $/ \mathrm{D}_{n}$-PAHs and PAHs/ $/ \mathrm{H}_{n}-\mathrm{PAHs}$, the assignment uncertainties of minor spectral features, or the uncertainties in the intrinsic intensities between different vibrational modes.
} 
K. D. Doney et al.: Deuterated polycyclic aromatic hydrocarbons: Revisited
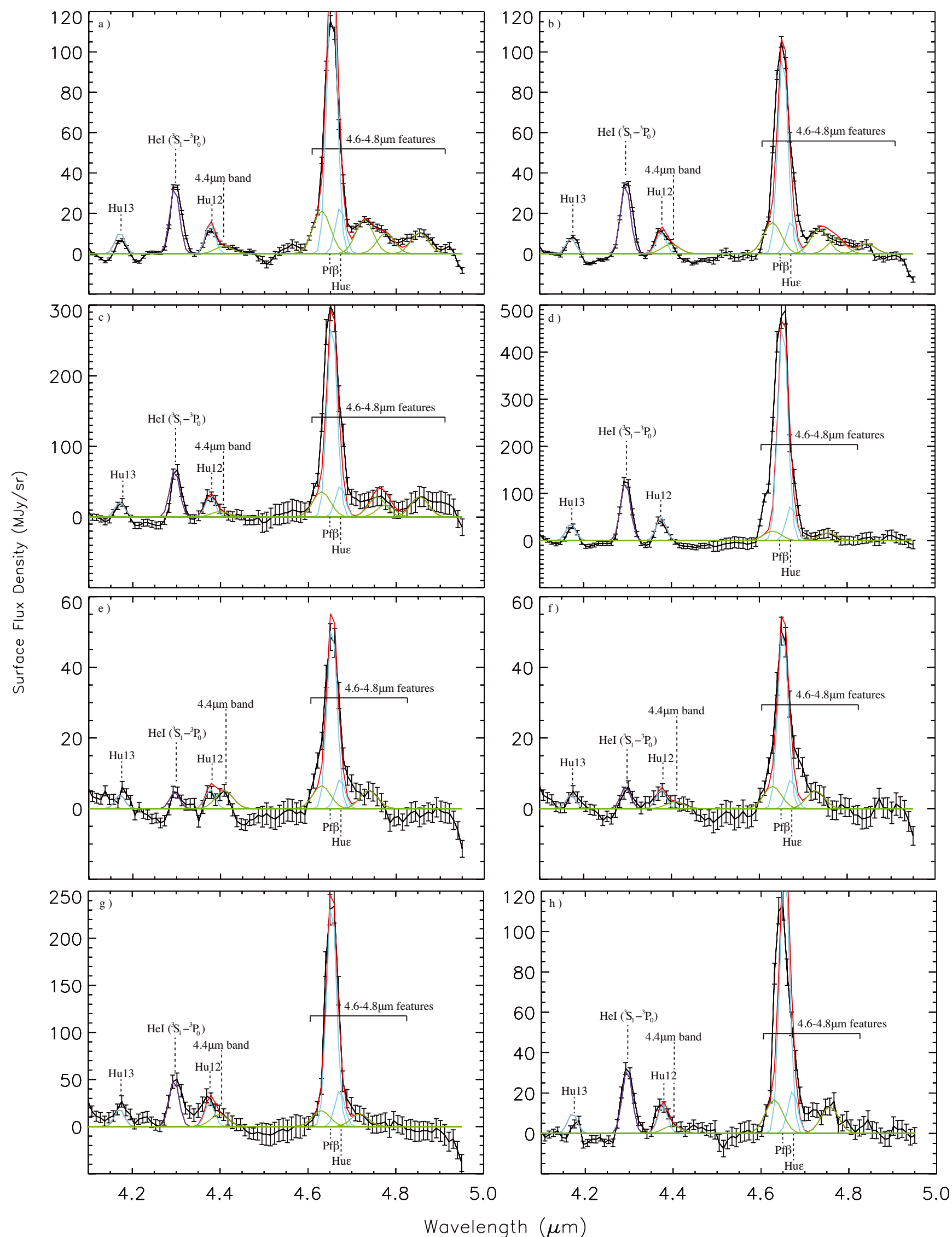

Fig. 2. Close up of the C-D stretching region of the spectra of a) IRAS 12073-6233 obs. 1; b) IRAS 12073-6233 obs. 2; c) NGC 3603; d) M17b; e) W51 obs. 1; f) W51 obs. 2; g) M8; and h) G75.783+0.343. The fitting results for the spectra is shown in red, the black line is the observed spectra, the $\mathrm{HI}$ emission lines are fit in cyan, the HeI emission line is fit in navy, and PAD and $\mathrm{D}_{n}$-PAH features are fit in green. 
$\mathrm{D} / \mathrm{H}$ ratio is consistent within flux uncertainties in W51, but not in IRAS 12073-6233. This may hint that very local conditions are indeed important to the deuterium variations, but more observations at higher spatial resolution are needed to confirm this.

The amount of deuterium at either an aromatic or aliphatic position was also determined through a comparison of observed $\mathrm{PAH} \mathrm{D} / \mathrm{H}$ ratios to those expected if one hydrogen is replaced with a deuterium at either an aromatic or aliphatic position. From the 3.4/3.29 $\mu \mathrm{m}$ ratio, the amount of aliphatic hydrogen relative to aromatic hydrogen, $N_{\text {aliphatic, }} / N_{\text {aromatic, } \mathrm{H}}$, of the PAHs in the eight observations was determined to be between $0.2-0.3$ (Table A.2), which is significantly larger than the $N_{\text {aliphatic,H }} / N_{\text {aromatic,H }} \sim 0.02$ typically seen in PAHs in the ISM (Tielens 2008). Additionally, neutral, small $\left(N_{C}<50\right)$ PAHs molecules are considered to mainly contribute to the $3.3 \mu \mathrm{m}$ feature, so from the PAH IR Spectral Database (Boersma et al. 2014) six representative PAHs (three extended and three compact) were chosen to determine the expected $\mathrm{D} / \mathrm{H}$ ratios: $\mathrm{C}_{40} \mathrm{H}_{22}$, $\mathrm{C}_{40} \mathrm{H}_{16}, \mathrm{C}_{32} \mathrm{H}_{18}, \mathrm{C}_{32} \mathrm{H}_{14}, \mathrm{C}_{24} \mathrm{H}_{14}$, and $\mathrm{C}_{24} \mathrm{H}_{12}$. For example, for $\mathrm{C}_{40} \mathrm{H}_{22}$ to get an $N_{\text {aliphatic,H}} / N_{\text {aromatic,H }} \sim 0.22$, there would be four aliphatic hydrogens and 18 aromatic hydrogens. Replacement of an aromatic hydrogen with an aromatic deuterium would result in an aromatic $\mathrm{D} / \mathrm{H}$ of 0.06 , and replacement of one aliphatic hydrogen for an aliphatic deuterium would result in an aliphatic $\mathrm{D} / \mathrm{H}$ ratio of 0.33 . If this is evaluated for the six representative molecules, on average a monodeuterated $\mathrm{D}_{n}-\mathrm{PAH}$ would have an expected aliphatic $\mathrm{D} / \mathrm{H}$ of $\sim 0.5$, while a monodeuteratued $\mathrm{PAD}$ would have an average expected aromatic $\mathrm{D} / \mathrm{H}$ of $\sim 0.09$.

The ratio of the $4.40 \mu \mathrm{m}$ features to the $3.29 \mu \mathrm{m}$ features gives a fractional abundance of aromatic deuterium to aromatic hydrogen of $<0.05$ for all eight observations. In contrast, based on the sum of the 4.63 and $4.75 \mu \mathrm{m}$ features relative to the sum of the 3.40 and $3.50 \mu \mathrm{m}$ features, the fractional abundance of aliphatic deuterium relative to aliphatic hydrogen is much larger, varying from 0.09 to 1 . For a representative PAH, for example, the hydrogenated $\mathrm{PAH} \mathrm{C}_{40} \mathrm{H}_{18}$, these observed deuterium abundances would yield at most 1 aromatic deuterium, 2 aliphatic deuterium, 13 aromatic hydrogen, and 2 aliphatic hydrogen, and a $\mathrm{PAH} \mathrm{D} / \mathrm{H}$ of 0.2 , which is roughly representative of the average of the values determined for the eight observations. Based on the expected $\mathrm{D} / \mathrm{H}$ ratios, it is estimated that in all eight observations less than $10 \%$ of the observed PAHs have one aromatic deuterium. Conversely, with the exception of the PAHs in W51 and M8, all of the observed PAHs have at least one aliphatic deuterium. In particular, the aliphatic $\mathrm{D} / \mathrm{H}$ ratios for IRAS 12073-6233 and M17b are more in agreement with the PAHs having one aliphatic deuterium for every aliphatic hydrogen. Furthermore, the aliphatic $\mathrm{D} / \mathrm{H}$ ratios $\sim 0.4$ and $\sim 0.30$ for IRAS $12073-6233$ obs. 1 and obs. 2, respectively, suggest that almost all of the deuterium is in aliphatic positions.

The determined amount of deuterium relative to hydrogen on PAHs are upper limits, and a more complete understanding would include the effects of the emission process on the band intensities; to understand these effects we calculated the emission spectrum of a prototype $\mathrm{PAH}$ molecule, neutral ovalene, where one solo hydrogen is substituted with a $-\mathrm{CH}_{2} \mathrm{D}$ group $\left(N_{\mathrm{C}}=33\right)$. To model the emission process, we follow the procedure described in Candian et al. (2012), where both the infrared spectrum of the molecule in question and its photoabsorption cross-section were evaluated with quantum chemistry techniques. As the effective temperature of the exciting source, we assume $T_{\text {eff }}=40000 \mathrm{~K}$, as in IRAS 12073-6233 (Martín-Hernández et al. 2002b), which is one of the HII regions in our sample.
For this molecule, the intrinsic 3.3/4.75 $\mu \mathrm{m}$ (C-H aromatic) $\mathrm{C}-\mathrm{D}$ aliphatic) ratio is 1.81 (consistent with earlier calculations of Bauschlicher et al. 1997). The emission process brings the band ratio to 0.64 , which then will correspond to a $\mathrm{D} / \mathrm{H}$ range of $0.01-0.16$ in our sample. These values are clearly sensitive to the parameters assumed in the emission model (e.g., effective temperature, PAH size), for example, M17, Peeters et al. (2004) did not consider the emission process and found a $\mathrm{D} / \mathrm{H}=$ $0.36 \pm 0.08$, while for the same source Onaka et al. (2014) via an emission model that favored larger PAHs found an upper limit $\mathrm{D} / \mathrm{H}=0.023 \pm 0.004$. Similarly, we find a $\mathrm{D} / \mathrm{H}=0.09 \pm 0.05$ for the nearby source M17b via our emission model, which favors smaller PAHs. Therefore, the observed band intensity ratio can overestimate the actual relative abundance of deuterium to hydrogen on PAHs and the amount is dependent on the emission process. Stronger constraints on the typical PAH size population and exciting source characteristics in HII regions would improve our estimate of the deuterium abundance.

\section{Discussion}

High levels of deuteration have been observed in some species (e.g., $\mathrm{CD}_{3} \mathrm{OH}, \mathrm{ND}_{3}$; Parise et al. 2004; Lis et al. 2002; van der Tak et al. 2002), which are thought to originate from grain surface chemistry (Roberts et al. 2003). Deuterium fractionation is not as extensive for PAHs. In the best case (IRAS 12073-6233 observation 1), the $\mathrm{PAH} \mathrm{D/H}$ value is 0.44 (Table A.3), which would translate to a fraction of gas-phase atomic deuterium (relative to hydrogen) locked up in PAHs of roughly $18 \mathrm{ppm}$; these are determined following the analysis method outlined in Onaka et al. (2014). Of the sources with observed deuterated PAHs, the average PAH D/H fraction is 0.21 , which corresponds to a locked up fraction of gas-phase deuterium (relative to hydrogen) of about $10 \mathrm{ppm}$. Observation of atomic deuterium in the local ISM shows strong variation in the $\mathrm{D} / \mathrm{H}$ abundance ratio of the gas phase at the $17 \mathrm{ppm}$ level (Linsky et al. 2006). While our observations are not along the same lines of sight, they indicate that PADs and $\mathrm{D}_{n}$-PAHs would be a major reservoir of elemental deuterium. Moreover, our study also reveals strong variation in the deuterated $\mathrm{PAH}$ to $\mathrm{PAH}$ ratio. Hence, the interaction of atomic deuterium with PAHs could well be at the origin of the observed variation in the gas-phase deuterium abundance.

While deuterated PAHs are not omnipresent, when present, deuteration is efficient; all eight observations have a PAH $\mathrm{D} / \mathrm{H}$ ratio that is significantly greater than the cosmic gas-phase abundance of $\sim 10^{-5}$ (Vidal-Madjar et al. 1998). Incorporation of deuterium into PAHs can occur through a number of mechanisms, most of which are driven by the small difference in zeropoint energy between hydrogen and deuterium. Deuterium enrichment can take place in the gas phase or through solid state reactions within ice or on grains.

Large deuteration fractionation can occur in PDRs at depths where most of the hydrogen is locked up in $\mathrm{H}_{2}$, but deuterium is still mainly atomic. $\mathrm{H}_{2}$ and $\mathrm{HD}$ are expected to show different behavior with depth into a cloud, as self-shielding of $\mathrm{H}_{2}$ pulls the $\mathrm{H} / \mathrm{H}_{2}$ transition to the surface of the PDR. Conversely, selfshielding is of little importance for HD, and photodestruction converts HD to atomic deuterium (Tielens 1992). Through gasphase addition reactions, the free atomic deuterium adds aliphatically to the PAH molecule. Recent theoretical calculations (Ricca et al. 2007; Rauls \& Hornekær 2008; Rasmussen et al. 2011) and experiments (Thrower et al. 2012; Klærke et al. 2013; 
Demarais et al. 2014) demonstrate that hydrogenation $\left(\mathrm{H}_{n}-\mathrm{PAH}\right)$ or deuteration $\left(\mathrm{D}_{n}-\mathrm{PAH}\right)$ can be important through reactions in the gas phase in regions of the PDR without intense UV radiation. The addition occurs preferentially on carbons at the edges of PAH molecules and gives the carbon an aliphatic character (Rauls \& Hornekær 2008). Rasmussen et al. (2011) and Rauls \& Hornekær (2008) calculated the first hydrogen addition to the periphery has a barrier of $0.06 \mathrm{eV}$ for neutral PAHs, while the second hydrogen addition is barrierless. For cations, hydrogenation is even easier, since the first hydrogen addition is barrierless and the second addition has a negligible barrier (Ricca et al. 2007). Similar mechanisms can be employed to explain the presence of aliphatic deuterium on PAHs. In an evaporating flow, the PAHs move to the surface of the PDR and then into the general ISM, during which the deuterium fractionation is temporarily preserved. In this schematic way, we can understand how PAHs can be highly fractionated, that this fractionation primarily occurs as aliphatic $\mathrm{H} / \mathrm{D}$, and that this fractionation behavior is very sensitive to the local conditions and history of the PAHs. Thus, it is expected to vary from one region to the next.

Additionally, ion-molecule and neutral-neutral addition reactions occur at low temperatures, but require that the reaction is exothermic or has no barrier (Dalgarno \& Lepp 1984; Tielens 1992; Bauschlicher 1998; Sandford et al. 2001). These reactions, in the gas phase or on grain surfaces, are proposed to lead to both aliphatic and aromatic deuterated or hydrogenated PAHs deep inside dense clouds,

$$
\begin{aligned}
& \mathrm{PAH}+\mathrm{H}_{2} \mathrm{D}^{+} \longrightarrow \mathrm{D}_{n}-\mathrm{PAH}^{+}+\mathrm{H}_{2} \\
& \mathrm{PAH}+\mathrm{H}_{3}^{+} \longrightarrow \mathrm{H}_{n}-\mathrm{PAH}^{+}+\mathrm{H}_{2} \\
& \mathrm{H}_{n}-\mathrm{PAD}^{+}+\mathrm{e}^{-} \longrightarrow \mathrm{PAD}+\mathrm{H} \\
& \mathrm{D}_{n}-\mathrm{PAH}^{+}+\mathrm{e}^{-} \longrightarrow \mathrm{PAH}+\mathrm{D} \\
& \mathrm{H}_{n}-\mathrm{PAH}^{+}+\mathrm{e}^{-} \longrightarrow \mathrm{PAH}+\mathrm{H} .
\end{aligned}
$$

Similar reaction schemes are responsible for the deuteration of small hydrocarbon species, such as $\mathrm{HCO}^{+}$and $\mathrm{HCN}$. Deuteration fractionation in these species is observed to reach levels of $\sim 4 \times 10^{-2}$ (Roberts et al. 2002).

At temperatures less than $50 \mathrm{~K}$, most of the volatile molecules are frozen out onto the dust grains (Boogert et al. 2015). Penetrating UV radiation from nearby $\mathrm{O} / \mathrm{B}$ stars or photon-induced, cosmic-ray ionization that is deep inside dense cores has enough energy to break the molecular bonds on smaller molecules producing radicals. These highly reactive species, in turn, can go on to form new bonds (Bernstein et al. 2001; Sandford et al. 2001). Laboratory experiments of PAHs in deuterium-enriched water ices demonstrated that under UV irradiations PAHs undergo oxidation, reduction, and deuteriumhydrogen exchange reactions. Deuterium enrichment in ices is independent of the size of the PAH, and seems to favor the aromatic deuterium product (PAD) over aliphatic addition, resulting in enrichment levels of at least $\mathrm{D} / \mathrm{H} \sim 10^{-4}$ (Sandford et al. 2000).

Independent of the temperature, PAHs can undergo unimolecular photodissociation if they absorb a UV photon with enough energy to break the $\mathrm{C}-\mathrm{H}$ bond. The aliphatic $\mathrm{sp}^{3}$ bonds are more labile compared to the aromatic $\mathrm{sp}^{2}$ bonds, favoring the loss of an aliphatic hydrogen or deuterium over an aromatic hydrogen or deuterium atom. The presence of aliphatic bonds also causes the PAH geometry to depart from planarity, adding stress to the molecule and resulting in weaker $\mathrm{C}-\mathrm{H}$ bonds than in fully aromatic, planar PAH molecules. The lower zero-point energy of deuterium suggests that dehydrogenation is favored over dedeuteration at ISM temperatures, $T<70 \mathrm{~K}$. In addition, since larger PAHs have larger heat capacities relative to small PAHs, unimolecular photodissociation favors PAHs with less than 50 carbon atoms. Theoretical estimates suggest that the expected deuterium fractionation from this method in HII regions is about $10^{-5}$ (Allamandola et al. 1989).

The low abundance or complete lack of observed deuterated features in a majority of the observed sources suggests that the conditions leading to deuterium addition in the ISM are not common. Further theoretical studies are required to assess the different scenarios in more detail.

\section{Conclusions}

Using AKARI, we searched for deuterated PAH emission in a sample of Galactic and extragalactic HII regions. We can conclude that

1. Deuterated PAHs have been observed in only six sources out of 41 in the Milky Way; this suggests that the incorporation of deuterium in PAHs is rare and highly dependent on the local conditions of the environment. The low $\mathrm{S} / \mathrm{N}$ in the spectrum of LMC and SMC HII regions prevented us from drawing conclusions about the relation between the $\mathrm{PAH} \mathrm{D/H}$ ratio and metallicity.

2. In our Galaxy, the average observed fractional abundance of deuterium relative to hydrogen locked up in PAHs is small, especially when compared with other interstellar molecules, such as $\mathrm{NH}_{3}$. Some sources show a PAH D/H ratio upper limit as high as 0.44 , or $\sim 0.2$ if emission process is considered. Thus, while PAHs do not appear to be the sole reservoir of deuterium, they can still explain part of the variation of the galactic gas-phase $\mathrm{D} / \mathrm{H}$.

3. Exclusion of the emission process in determining band intensities can overestimate the abundance of deuterium relative to hydrogen locked up in PAHs. The exact magnitude of this effect is found to be strongly dependent on both the size of the PAH and the characteristics of the exciting source.

4. The PAHs are observed to be deuterium enriched relative to the galactic gas-phase abundance, since the deuterium atom is preferentially added to an aliphatic position.

The upcoming James Webb Space Telescope (JWST) will offer significantly better spectral resolution in the NIR, from $1-5 \mu \mathrm{m}$, which will allow for better resolution of the 4.63 and $4.75 \mu \mathrm{m}$ features, and even better constraint on the abundance of deuterium on PAHs, in the Milky Way, and in the neighboring LMC and SMC. Additionally, JWST will offer similar resolution MIR spectroscopy from 5-28 $\mu \mathrm{m}$, which gives access to the intrinsically stronger $\mathrm{C}-\mathrm{D}$ bending modes in addition to the C-D stretch modes. Since the C-D bending features are hard to unambiguously distinguish from the $\mathrm{C}-\mathrm{H}$ bending features of PAH cations, the simultaneous detection of the C-D bending and C-D stretching features is needed for a stronger confirmation that deuterated PAHs were detected; this is a unique capability of JWST, which previous telescopes, for example, the Spitzer Space Telescope, were not able to do.

Acknowledgements. We would like to give a special thanks to the referee whose advice greatly helped the clarity of the paper. K.D.D. thanks Dr. E. Peeters for useful and stimulating discussions. This research is based on observations with AKARI, a JAXA project with the participation of ESA. Studies of interstellar PAHs at Leiden Observatory are supported through advanced European Research Council grant 246976 and a Spinoza award. 


\section{References}

Allamandola, L. J. 1993, in Astronomical Infrared Spectroscopy: Future Observational Directions, ed. S. Kwok, ASP Conf. Ser., 41, 197

Allamandola, L. J., Tielens, A. G. G. M., \& Barker, J. R. 1985, ApJ, 290, L25 Allamandola, L. J., Tielens, A. G. G. M., \& Barker, J. R. 1989, ApJS, 71, 733 Bauschlicher, Jr., C. W. 1998, ApJ, 509, L125

Bauschlicher, Jr., C. W., Langhoff, S. R., Sandford, S. A., \& Hudgins, D. M. 1997, J. Phys. Chem. A, 101, 2414

Bernstein, M. P., Dworkin, J. P., Sandford, S. A., \& Allamandola, L. J. 2001, Meteorit. Planet. Sci., 36, 351

Bertoldi, F., Draine, B. T., Rosenthal, D., et al. 2000, in From Molecular Clouds to Planetary, eds. Y. C. Minh, \& E. F. van Dishoeck, IAU Symp., 197, 191

Boersma, C., Bauschlicher, Jr., C. W., Ricca, A., et al. 2014, ApJS, 211, 8

Boogert, A., Gerakines, P., \& Whittet, D. 2015, ARA\&A, 53, 541

Buragohain, M., Pathak, A., Sarre, P., Onaka, T., \& Sakon, I. 2015, MNRAS, 454, 193

Candian, A., Kerr, T. H., Song, I.-O., McCombie, J., \& Sarre, P. J. 2012, MNRAS, 426, 389

Cherchneff, I., Barker, J. R., \& Tielens, A. G. G. M. 1992, ApJ, 401, 269

Dalgarno, A., \& Lepp, S. 1984, ApJ, 287, L47

Demarais, N. J., Yang, Z., Snow, T. P., \& Bierbaum, V. M. 2014, ApJ, 784, 25

Draine, B. T. 2004, ArXiv e-prints [arXiv: astro-ph/0410310]

Epstein, R. I., Lattimer, J. M., \& Schramm, D. N. 1976, Nature, 263, 198

Filipovic, M. D., Jones, P. A., White, G. L., \& Haynes, R. F. 1998, A\&AS, 130, 441

Frenklach, M., \& Feigelson, E. D. 1989, ApJ, 341, 372

Gerakines, P. A., Schutte, W. A., \& Ehrenfreund, P. 1996, A\&A, 312, 289

Hilditch, R. W., Howarth, I. D., \& Harries, T. J. 2005, MNRAS, 357, 304

Hudgins, D. M., Bauschlicher, Jr., C. W., \& Sandford, S. A. 2004, ApJ, 614, 770

Joblin, C., \& Tielens, A. G. G. M., eds. 2011, PAHs and the Universe: A Symposium to Celebrate the 25th Anniversary of the PAH Hypothesis, EAS Pub. Ser., 46

Kerridge, J. F., Chang, S., \& Shipp, R. 1987, Geochim. Cosmochim. Acta, 51, 2527

Klærke, B., Toker, Y., Rahbek, D. B., Hornekær, L., \& Andersen, L. H. 2013, A\&A, 549, A84
Leger, A., \& Puget, J. L. 1984, A\&A, 137, L5

Li, A., \& Draine, B. T. 2012, ApJ, 760, L35

Linsky, J. L., Draine, B. T., Moos, H. W., et al. 2006, ApJ, 647, 1106

Lis, D. C., Roueff, E., Gerin, M., et al. 2002, ApJ, 571, L55

Martín-Hernández, N. L., Peeters, E., Morisset, C., et al. 2002a, A\&A, 381, 606

Martín-Hernández, N. L., Vermeij, R., Tielens, A. G. G. M., van der Hulst, J. M., \& Peeters, E. 2002b, A\&A, 389, 286

Mori, T. I., Onaka, T., Sakon, I., et al. 2014, ApJ, 784, 53

Onaka, T., Matsuhara, H., Wada, T., et al. 2007, PASJ, 59, 401

Onaka, T., Lorente, R., \& Ita, Y. 2009, IRC Data Users's Manual for Phase 3 ver 1.1

Onaka, T., Mori, T. I., Sakon, I., et al. 2014, ApJ, 780, 114

Paladini, R., Burigana, C., Davies, R. D., et al. 2003, A\&A, 397, 213

Parise, B., Castets, A., Herbst, E., et al. 2004, A\&A, 416, 159

Peeters, E., Allamandola, L. J., Bauschlicher, Jr., C. W., et al. 2004, ApJ, 604, 252

Pietrzyński, G., Graczyk, D., Gieren, W., et al. 2013, Nature, 495, 76

Pilleri, P., Joblin, C., Boulanger, F., \& Onaka, T. 2015, A\&A, 577, A16

Rasmussen, J. A., Henkelman, G., \& Hammer, B. 2011, J. Chem. Phys., 134, 164703

Rauls, E., \& Hornekær, L. 2008, ApJ, 679, 531

Ricca, A., Bakes, E. L. O., \& Bauschlicher, Jr., C. W. 2007, ApJ, 659, 858

Roberts, H., Fuller, G. A., Millar, T. J., Hatchell, J., \& Buckle, J. V. 2002, Planet. Space Sci., 50, 1173

Roberts, H., Herbst, E., \& Millar, T. J. 2003, ApJ, 591, L41

Sandford, S. A., Bernstein, M. P., Allamandola, L. J., Gillette, J. S., \& Zare, R. N. 2000, ApJ, 538, 691

Sandford, S. A., Bernstein, M. P., \& Dworkin, J. P. 2001, Meteor. Planet. Sci., 36,1117

Sandford, S. A., Bernstein, M. P., \& Materese, C. K. 2013, ApJS, 205, 8

Schutte, W. A., Tielens, A. G. G. M., \& Allamandola, L. J. 1993, ApJ, 415, 397

Storey, P. J., \& Hummer, D. G. 1995, MNRAS, 272, 41

Thrower, J. D., Jørgensen, B., Friis, E. E., et al. 2012, ApJ, 752, 3

Tielens, A. G. G. M. 1992, in Astrochemistry of Cosmic Phenomena, ed. P. D. Singh, IAU Symp., 150, 91

Tielens, A. G. G. M. 2008, ARA\&A, 46, 289

van der Tak, F. F. S., Schilke, P., Müller, H. S. P., et al. 2002, A\&A, 388, L53

Vidal-Madjar, A., Lemoine, M., Ferlet, R., et al. 1998, A\&A, 338, 694 
K. D. Doney et al.: Deuterated polycyclic aromatic hydrocarbons: Revisited

\section{Appendix A: Additional tables}

Table A.1. Observation log and target parameters.

\begin{tabular}{|c|c|c|c|c|}
\hline \multirow[t]{2}{*}{ Target } & \multicolumn{2}{|c|}{ Slit center position ${ }^{a}$} & \multirow[t]{2}{*}{ Obs. ID } & \multirow[t]{2}{*}{ Obs. date } \\
\hline & RA & Dec. & & \\
\hline LMCN4A & 73.029 & -66.921 & $4300021.1,4300021.2,4301021.1$ & 2008 Dec. 4, 2008 Dec. 4, 2009 Dec. 4 \\
\hline LMCN83B & 73.609 & -69.184 & $4300022.1,4300022.2,4301022.1$ & 2008 Nov. 14, 2008 Nov. 14,2009 Nov. 18 \\
\hline LMCN57A & 83.104 & -67.698 & $4300023.1,4300023.2,4301023.2$ & 2008 Nov. 8, 2008 Nov. 8, 2009 Nov. 8 \\
\hline LMCN105A-IRS1 & 77.453 & -68.879 & $4300024.1,4300024.2,4301024.2$ & 2008 Nov. 11, 2008 Nov. 11, 2009 Nov. 12 \\
\hline LMCN91A & 74.313 & -68.442 & $4300025.1,4300025.2,4301025.3$ & 2008 Nov. 19,2008 Nov. 20,2009 Nov. 24 \\
\hline LMCN77A & 72.363 & -69.202 & $4300026.1,4300026.2,4301026.3$ & 2008 Nov. 16, 2008 Nov. 16, 2009 Nov. 18 \\
\hline LMCN191A & 76.157 & -70.908 & $4300027.1,4300027.2,4301027.4$ & 2008 Oct. 28,2008 Oct. 28,2009 Nov. 6 \\
\hline SMCN26 & 12.036 & -73.249 & $4300028.1,4300028.2$ & 2008 Nov. 1, 2008 Nov. 2 \\
\hline SMCN10 & 11.235 & -73.170 & $4300029.1,4300029.2$ & 2008 Oct. 31,2008 Nov. 1 \\
\hline SMCN88A & 21.033 & -73.151 & $4300030.1,4300030.2$ & 2008 Nov. 4, 2008 Nov. 4 \\
\hline SMCN66 & 14.772 & -72.177 & 4300031.1, 4300031.2, 4301031.1 & 2008 Nov. 4, 2008 Nov. 5, 2009 Nov. 6 \\
\hline SMCN81 & 17.304 & -73.194 & $4300032.1,4300032.2$ & 2008 Nov. 3, 2008 Nov. 3 \\
\hline IRAS $14567-5846$ & 225.230 & -58.981 & $4300033.1,4300033.2$ & 2009 Feb. 19, 2009, Feb. 19 \\
\hline IRAS 15384-5348 & 235.569 & -53.976 & $4300034.1,4300034.2$ & 2009 Feb. 24, 2009 Feb. 24 \\
\hline IRAS 15502-5302 & 238.527 & -53.194 & $4300035.1,4300035.2$ & 2009 Feb. 26, 2009 Feb. 26 \\
\hline IRAS 12073-6233 & 182.494 & -62.832 & $4300036.1,4300036.2$ & 2009 Jan. 28, 2009 Jan. 28 \\
\hline GAL314.2+00.3 & 216.237 & -60.511 & $4300037.1,4300037.2$ & 2009 Feb. 14, 2009 Aug. 19 \\
\hline GAL319.9+00.8 & 225.905 & -57.650 & $4300038.1,4300038.2$ & 2009 Feb. 19, 2009 Feb. 19 \\
\hline GAL336.0+00.1 & 247.744 & -48.164 & $4300039.1,4300039.2$ & 2009 Mar. 3, 2009 Mar. 3 \\
\hline GAL334.7-00.7 & 247.269 & -49.656 & $4300040.1,4300040.2$ & 2009 Mar. 3, 2009 Mar. 4 \\
\hline M8 & 270.922 & -24.377 & 5200161.1 & 2008 Sep. 23 \\
\hline G8.137+0.228 & 270.759 & -21.800 & 5200163.1 & 2008 Sep. 22 \\
\hline W31a & 272.363 & -20.322 & 5200165.1 & 2008 Sep. 24 \\
\hline W31b & 272.255 & -20.084 & 5200167.1 & 2008 Sep. 24 \\
\hline M $17 b$ & 275.119 & -16.204 & 5200171.1 & 2008 Sep. 28 \\
\hline M 17a & 275.110 & -16.181 & 5200169.1 & 2008 Sep. 27 \\
\hline W42 & 279.564 & -6.795 & $5200294.1,5200294.2$ & 2008 Oct. 2,2008 Oct. 2 \\
\hline G29.944-0.042 & 281.518 & -2.653 & $5200295.1,5200295.2$ & 2008 Oct. 4, 2008 Oct. 4 \\
\hline W49A & 287.568 & 9.108 & $5200299.1,5200299.2$ & 2008 Oct. 12,2007 Oct. 13 \\
\hline G48.596+0.042 & 290.127 & 13.930 & $5200300.1,5200300.2$ & 2008 Oct. 17,2008 Oct. 17 \\
\hline W51 & 290.561 & 14.051 & $5200301.1,5200301.2$ & 2008 Oct. 17,2008 Oct. 18 \\
\hline W58A & 300.440 & 33.548 & 5201198.1, 5200767.1 & 2009 May 2, 2009 Nov. 6 \\
\hline G70.293+1.600 & 300.440 & 33.548 & 5200337.1 & 2008 Nov. 6 \\
\hline G75.783+0.343 & 305.422 & 37.430 & 5200772.1 & 2009 May 11 \\
\hline G76.383-0.621 & 306.863 & 37.381 & 5200343.1 & 2008 Nov. 15 \\
\hline G78.438+2.659 & 304.913 & 40.943 & 5200776.1 & 2009 May 13 \\
\hline DR7 & 307.037 & 40.875 & 5200769.1 & 2009 May 17 \\
\hline G81.679+0.537 & 309.752 & 42.331 & 5200347.1 & 2008 Nov. 22 \\
\hline G111.282-0.663 & 349.020 & 60.038 & 5200432.1 & 2009 Jan. 16 \\
\hline RCW42 & 141.106 & -51.990 & 5200452.1 & 2008 Dec. 15 \\
\hline G282.023-1.180 & 151.653 & -57.204 & 5200436.1 & 2009 Jan. 1 \\
\hline RCW49 & 156.034 & -57.788 & 5200438.1 & 2009 Jan. 4 \\
\hline NGC 3372 & 160.883 & -59.580 & 5200440.1 & 2009 Jan. 10 \\
\hline G289.066-0.357 & 164.124 & -60.098 & 5200442.1 & 2009 Jan. 13 \\
\hline NGC 3576 & 167.984 & -61.313 & 5200444.1 & 2009 Jan. 17 \\
\hline NGC 3603 & 168.756 & -61.263 & 5200446.1 & 2009 Jan. 17 \\
\hline G319.158-0.398 & 225.816 & -59.074 & 5200933.1 & 2009 Aug. 25 \\
\hline G330.868-0.365 & 242.601 & -52.099 & 5200109.1 & 2008 Sep. 2 \\
\hline G331.386-0.359 & 243.183 & -51.748 & 5200113.1 & 2008 Sep. 3 \\
\hline G333.122-0.446 & 245.255 & -50.585 & 5200121.1 & 2008 Sep. 4 \\
\hline G338.398+0.164 & 250.032 & -46.385 & $5200942.1,5200942.2$ & 2009 Sep. 7, 2009 Sep. 7 \\
\hline G338.400-0.201 & 250.468 & -46.582 & 5200943.2 & 2009 Sep. 7 \\
\hline G345.528-0.051 & 256.538 & -40.962 & 5200133.1 & 2008 Sep. 11 \\
\hline
\end{tabular}

Notes. ${ }^{(a)}$ Intended AKARI target position in degrees, J2000. 
A\&A 586, A65 (2016)

Table A.2. PAH/ $\mathrm{H}_{n}-\mathrm{PAH}$ fluxes for sources with detectable deuterated features.

\begin{tabular}{|c|c|c|c|c|c|c|}
\hline Source & Av (mag) & $\begin{array}{l}3.29 \mu \mathrm{m} \\
\text { Aromatic } \mathrm{CH}\end{array}$ & $\begin{array}{l}3.4 \mu \mathrm{m} \\
\text { Asymmetric } \\
\text { Aliphatic } \mathrm{CH}\end{array}$ & $3.45 \mu \mathrm{m}^{a}$ & $\begin{array}{l}3.5 \mu \mathrm{m} \\
\text { Symmetric } \\
\text { Aliphatic CH }\end{array}$ & $3.56 \mu \mathrm{m}^{a}$ \\
\hline G75.783+0.343 & 28 & $\begin{array}{l}22.2 \pm 2.0 \\
125 \sigma\end{array}$ & $\begin{array}{l}6.2 \pm 0.6 \\
37 \sigma\end{array}$ & $\begin{array}{l}3.3 \pm 0.4 \\
20 \sigma\end{array}$ & $\begin{array}{l}2.9 \pm 0.9 \\
18 \sigma\end{array}$ & $\begin{array}{l}0.9 \pm 0.2 \\
5.5 \sigma\end{array}$ \\
\hline NGC 3603 & 22 & $\begin{array}{l}25.1 \pm 2.4 \\
69 \sigma\end{array}$ & $\begin{array}{l}7.8 \pm 0.8 \\
23 \sigma\end{array}$ & $\begin{array}{l}4.6 \pm 0.6 \\
14 \sigma\end{array}$ & $\begin{array}{l}3.1 \pm 1.2 \\
9.5 \sigma\end{array}$ & $\begin{array}{l}1.2 \pm 0.5 \\
3.7 \sigma\end{array}$ \\
\hline W51 obs. 1 & 26 & $\begin{array}{l}20.0 \pm 1.8 \\
182 \sigma\end{array}$ & $\begin{array}{l}4.8 \pm 0.5 \\
47 \sigma\end{array}$ & $\begin{array}{l}3.2 \pm 0.3 \\
32 \sigma\end{array}$ & $\begin{array}{l}2.3 \pm 0.5 \\
23 \sigma\end{array}$ & $\begin{array}{l}0.9 \pm 0.2 \\
9.5 \sigma\end{array}$ \\
\hline W51 obs. 2 & 26 & $\begin{array}{l}20.6 \pm 1.8 \\
232 \sigma\end{array}$ & $\begin{array}{l}4.9 \pm 0.5 \\
59 \sigma\end{array}$ & $\begin{array}{l}3.2 \pm 0.3 \\
39 \sigma\end{array}$ & $\begin{array}{l}2.2 \pm 0.4 \\
27 \sigma\end{array}$ & $\begin{array}{l}1.2 \pm 0.2 \\
15 \sigma\end{array}$ \\
\hline M17b & 23 & $\begin{array}{l}12.3 \pm 1.5 \\
20 \sigma\end{array}$ & $\begin{array}{l}2.9 \pm 0.5 \\
5.1 \sigma\end{array}$ & $\begin{array}{l}2.1 \pm 0.4 \\
3.6 \sigma\end{array}$ & $\begin{array}{l}0.8 \pm 0.5 \\
1.5 \sigma\end{array}$ & $\begin{array}{l}0.8 \pm 0.4 \\
1.4 \sigma\end{array}$ \\
\hline M8 & 16 & $\begin{array}{l}99.1 \pm 8.9 \\
175 \sigma\end{array}$ & $\begin{array}{l}22.9 \pm 2.2 \\
44 \sigma\end{array}$ & $\begin{array}{l}14.7 \pm 1.5 \\
28 \sigma\end{array}$ & $\begin{array}{l}12.1 \pm 2.2 \\
24 \sigma\end{array}$ & $\begin{array}{l}5.5 \pm 0.9 \\
11 \sigma\end{array}$ \\
\hline IRAS $12073-6233$ obs. 1 & 31 & $\begin{array}{l}11.7 \pm 1.0 \\
149 \sigma\end{array}$ & $\begin{array}{l}2.9 \pm 0.3 \\
40 \sigma\end{array}$ & $\begin{array}{l}1.9 \pm 0.2 \\
27 \sigma\end{array}$ & $\begin{array}{l}1.3 \pm 0.2 \\
18 \sigma\end{array}$ & $\begin{array}{l}0.6 \pm 0.1 \\
9.0 \sigma\end{array}$ \\
\hline IRAS $12073-6233$ obs. 2 & 22 & $\begin{array}{l}12.2 \pm 1.0 \\
69 \sigma\end{array}$ & $\begin{array}{l}2.6 \pm 0.2 \\
16 \sigma\end{array}$ & $\begin{array}{l}1.7 \pm 0.2 \\
11 \sigma\end{array}$ & $\begin{array}{l}1.3 \pm 0.2 \\
8.0 \sigma\end{array}$ & $\begin{array}{l}0.5 \pm 0.1 \\
2.8 \sigma\end{array}$ \\
\hline
\end{tabular}

Notes. In units of $10^{-17} \mathrm{Wm}^{-2} \operatorname{arcsec}^{-2}$. ${ }^{(a)}$ See text for discussion of the origin of these features.

Table A.3. PAD/D $-\mathrm{PAH}$ fluxes for sources with detectable deuterated features.

\begin{tabular}{|c|c|c|c|c|c|c|}
\hline Source & $\begin{array}{l}4.4 \mu \mathrm{m} \\
\text { Aromatic CD }\end{array}$ & $\begin{array}{l}4.63 \mu \mathrm{m} \\
\text { Asymmetric } \\
\text { Aliphatic CD }\end{array}$ & $\begin{array}{l}4.75 \mu \mathrm{m} \\
\text { Symmetric } \\
\text { Aliphatic CD }\end{array}$ & $4.8 \mu \mathrm{m}^{a}$ & $4.85 \mu \mathrm{m}^{a}$ & $\mathrm{D} / \mathrm{H}^{b}$ \\
\hline $\mathrm{G} 75.783+0.343$ & $\begin{array}{l}0.30 \pm 0.19 \\
1.2 \sigma\end{array}$ & $\begin{array}{l}1.26 \pm 0.53 \\
5.3 \sigma\end{array}$ & $\begin{array}{l}0.99 \pm 0.33 \\
4.2 \sigma\end{array}$ & & & $0.13 \pm 0.03$ \\
\hline NGC 3603 & $\begin{array}{l}0.62 \pm 0.52 \\
1.3 \sigma\end{array}$ & $\begin{array}{l}2.73 \pm 1.57 \\
5.8 \sigma\end{array}$ & $\begin{array}{l}2.13 \pm 0.98 \\
4.7 \sigma\end{array}$ & $\begin{array}{l}1.73 \pm 0.79 \\
3.8 \sigma\end{array}$ & $\begin{array}{l}2.11 \pm 1.07 \\
4.7 \sigma\end{array}$ & $0.37 \pm 0.10$ \\
\hline W51 obs. 1 & $\begin{array}{l}0.41 \pm 0.14 \\
5.4 \sigma\end{array}$ & $\begin{array}{l}0.49 \pm 0.29 \\
6.8 \sigma\end{array}$ & $\begin{array}{l}0.39 \pm 0.18 \\
5.5 \sigma\end{array}$ & & & $0.07 \pm 0.02$ \\
\hline W51 obs. 2 & $\begin{array}{l}0.16 \pm 0.14 \\
2.1 \sigma\end{array}$ & $\begin{array}{l}0.48 \pm 0.29 \\
6.6 \sigma\end{array}$ & $\begin{array}{l}0.38 \pm 0.18 \\
5.3 \sigma\end{array}$ & & & $0.06 \pm 0.02$ \\
\hline M17b & & $\begin{array}{l}1.53 \pm 1.19 \\
6.6 \sigma\end{array}$ & $\begin{array}{l}1.19 \pm 0.75 \\
5.3 \sigma\end{array}$ & & & $0.25 \pm 0.13$ \\
\hline M8 & $\begin{array}{l}1.03 \pm 0.53 \\
4.5 \sigma\end{array}$ & $\begin{array}{l}1.05 \pm 1.00 \\
4.8 \sigma\end{array}$ & $\begin{array}{l}0.82 \pm 0.63 \\
3.8 \sigma\end{array}$ & & & $0.03 \pm 0.01$ \\
\hline IRAS $12073-6233$ obs. 1 & $\begin{array}{l}0.30 \pm 0.08 \\
1.2 \sigma\end{array}$ & $\begin{array}{l}1.62 \pm 0.28 \\
6.7 \sigma\end{array}$ & $\begin{array}{l}1.26 \pm 0.17 \\
5.3 \sigma\end{array}$ & $\begin{array}{l}0.76 \pm 0.15 \\
3.2 \sigma\end{array}$ & $\begin{array}{l}0.76 \pm 0.16 \\
3.3 \sigma\end{array}$ & $0.44 \pm 0.05$ \\
\hline IRAS $12073-6233$ obs. 2 & $\begin{array}{l}0.37 \pm 0.08 \\
3.2 \sigma\end{array}$ & $\begin{array}{l}1.18 \pm 0.23 \\
10.7 \sigma\end{array}$ & $\begin{array}{l}0.92 \pm 0.23 \\
8.5 \sigma\end{array}$ & $\begin{array}{l}0.46 \pm 0.16 \\
4.3 \sigma\end{array}$ & $\begin{array}{l}0.36 \pm 0.14 \\
3.4 \sigma\end{array}$ & $0.31 \pm 0.04$ \\
\hline
\end{tabular}

Notes. In units of $10^{-17} \mathrm{Wm}^{-2} \operatorname{arcsec}^{-2}$. ${ }^{(a)}$ See text for discussion of the origin of these features. ${ }^{(b)}$ The PAH D/H is calculated as the sum of the deuterium feature fluxes (Table A.3 Cols. 2-6) divided by a factor of 1.75 to account for intrinsic intensities, divided by the sum of the hydrogen feature fluxes (Table A.2 Cols. 3-7). The quoted uncertainties are based on the flux uncertainties alone. 